\title{
ANÁLISE ESTRUTURAL DE UMA TURBINA EÓLICA DE EIXO VERTICAL
}

\author{
Glaylson Silva Craveiro e Francisco Frederico dos Santos Matos \\ Instituto Federal de Educação, Ciência e Tecnologia do Ceará, Campus de Maracanaú \\ Programa de Pós-graduação em Energias Renováveis (PPGER) \\ <glaylsoncraveiro@gmail.com>,<francisco.f.matos@gmail.com>
}

DOI: $10.21439 /$ conexoes.v10i5.1165

\begin{abstract}
Resumo. A demanda crescente por energia no mundo tem gerado cada vez mais pesquisa e desenvolvimento na área de energias renováveis. Dentre as mais abordadas está a energia eólica, a qual tem sido transformada em energia elétrica predominantemente por turbinas eólicas de eixo horizontal (TEEH). Contudo, evidenciam-se escassos estudos relacionados às turbinas eólicas de eixo vertical (TEEV). Nesse raciocínio, iniciou-se uma atividade objetivando a análise estrutural de uma TEEV com um modelo de instalação previamente idealizado do tipo Darrieus no meio urbano. Foi traçado um estudo dos materiais utilizados, buscando eliminar ou, pelo menos, minimizar adversidades estruturais. Tal modelo foi desenvolvido inicialmente em uma plataforma de desenho computacional 3D. Nesta etapa de criação do modelo, buscou-se fazer o uso do mínimo de material possível de forma que a leveza da turbina fosse preservada. O modelo foi exportado para o Ansys (2012), onde foi dada sequência à simulação estrutural estática por elementos finitos. Foram realizadas simulações aplicando a rotação nominal da turbina, incluindo outras condições de contorno a fim de verificar os fatores de segurança mínimos e deformações sofridas. Através da análise dos resultados, foram propostas pequenas alterações na geometria, afim de chegar em um modelo satisfatório.
\end{abstract}

Palavras-chaves: Darrieus, energia eólica, simulação estrutural.

\begin{abstract}
The growing demand for energy in the world has generated more and more research and development in the field of renewable energy. Among the most discussed are wind energy, which has been transformed into electric power predominantly by horizontal axis wind turbines (TEEH). However, there are few studies related to vertical axis turbines (TEEV). In this reasoning, an activity was started aiming at a structural analysis of a TEEV with a previously idealized model of the Darrieus type in the urban environment. A study of the materials used was designed to eliminate or minimize structural adversities. Such a model was initially developed on a 3D computer design platform. In this stage of creation of the model, we tried to make use of the minimum of material possible so that a lightness of the turbine was preserved. The model was exported to Ansys (2012), where the finite element static structural simulation was given. Structural simulations were performed applying nominal turbine rotation, including other boundary conditions to verify the minimum safety factors and deformations suffered. Trough the result analysis, small changes were proposed in the geometry, in order to arrive at a satisfactory model.
\end{abstract}

Keywords: Darrieus, wind power, structural simulation.

\section{INTRODUÇÃO}

O mundo atualmente necessita da utilização de fontes renováveis de energia, como energia solar, eólica, geotérmica, dentre outras, como aponta em Kennedy (2005). No entanto, a matriz energética brasileira, segundo a Empresa de Pesquisa Energética (EPE, 2015), é praticamente baseada em fontes provenientes de hidrelétricas, em virtude da abundância de água em boa parte do território nacional. No entanto, conforme Martins, Guarnieri e Pereira (2008), já verifica-se que a energia eólica está entre as mais abordadas em pesquisas sobre energias renováveis, destacando-se com um ritmo cres- 
cente de investimento.

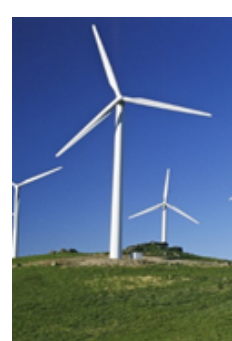

(a)

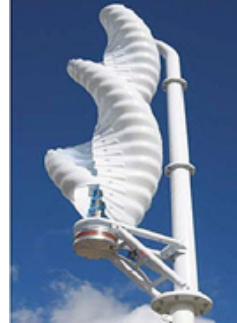

(b)

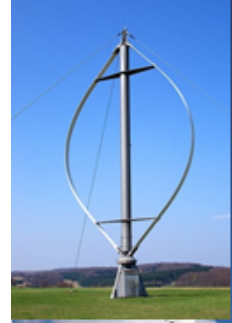

(c)
Figura 1: a)Turbina eólica de eixo horizontal; b) Turbina eólica do tipo Savonius; c) Turbina eólica de eixo vertical do tipo Darrieus Fonte: Braga et al. (2012).

As turbinas eólicas podem ser classificadas a partir da orientação do seu eixo de rotação, tais como: turbina eólica de eixo horizontal (TEEH), ilustrada na Fig 11a e turbina eólica de eixo vertical (TEEV), Figs $1 \mathrm{~b}$ e 1 c. Os projetos que utilizam as TEEHs foram, ao longo do tempo, melhorados, possibilitando até a sua instalação em grandes plataformas marítimas. Por outro lado, as TEEVs, por problemas de vibração excessiva e por apresentarem baixa eficiência energética em relação as TEEHs, tiveram o seu desenvolvimento interrompido em meados da década de 80 (HOWELL et al., 2010).

Muitos estudos, como o de Leandro Filho, Silva Junior e Duarte Junior (2012), utilizam de métodos numéricos para aperfeiçoar e verificar a segurança de estruturas como uma estimativa inicial, antes de recorrer à análise experimental. Dentre os mais utilizados está o método de elementos finitos, conforme cita Awruch e Gomes (2014), substituindo uma análise experimental similar, como realizado em Li et al. (2016).

Baseado na pesquisa de Fagan et al. (2017), Farenzena, Carrazedo e Baptista Junior (2012) e Leandro Filho, Silva Junior e Duarte Junior (2012), o presente projeto tem como objetivo a avaliação estrutural de uma TEEV (tipo Darrieus) de pequeno porte, a fim de analisar sua estrutura por meio de um software de simulação computacional (ANSYS, 2012). Nesse sentido, e ainda visando uma turbina de baixo custo e leveza, o estudo foi direcionado ao campo de previsão e análise de falhas estruturais, resultando em pequenas modificações da geometria inicial.

\section{METODOLOGIA}

A turbina proposta utiliza materiais que atendam às necessidades de geração de energia, com o menor custo envolvido e geometria de fácil montagem. Para tanto, foi realizado uma seleção dos materiais, buscando eli- minar ou minimizar problemas estruturais. Inicalmente, o modelo foi desenvolvido em uma plataforma de modelagem 3D, o Inventor (2016), onde nessa etapa de criação buscou-se utilizar o mínimo de material para garantir a leveza necessária e a fixação dos componentes da turbina. Na Tab11 é apresentada a descrição dos principais componentes da turbina, onde também são observados na Fig 2 .

Tabela 1: Principais componentes da turbina e a sua funcionalidade

\begin{tabular}{|c|c|}
\hline Componentes & Material e Função \\
\hline 1 - Eixo Central & $\begin{array}{l}\text { Componente de aço fixado } \\
\text { a torre da turbina, utilizado } \\
\text { para fixar o estator e o rotor }\end{array}$ \\
\hline 2 - Estator & $\begin{array}{l}\text { Componente de nylon para } \\
\text { fixação das bobinas de cobre }\end{array}$ \\
\hline 3 e 4 - Rolamentos & $\begin{array}{l}\text { Componentes monta- } \\
\text { dos na parte interna do } \\
\text { tubo flange para a fixa- } \\
\text { ção com o eixo central }\end{array}$ \\
\hline 5 - Tubo Flange & $\begin{array}{l}\text { Componente de nylon } \\
\text { para a fixação dos suporte } \\
\text { das pás e dos rolamentos }\end{array}$ \\
\hline 6 - Tampa & $\begin{array}{l}\text { Componente de nylon } \\
\text { para evitar o contato da } \\
\text { chuva e poeira com o } \\
\text { eixo central e rolamentos }\end{array}$ \\
\hline 7 - Suporte & $\begin{array}{l}\text { Componente estrutural de } \\
\text { alumínio para a conexão } \\
\text { entre o tubo flange e as pás }\end{array}$ \\
\hline 8 - Pás & $\begin{array}{l}\text { Componente aerodinâ- } \\
\text { mico de madeira de balsa } \\
\text { e alúmínio para promo- } \\
\text { ver a rotação da turbina }\end{array}$ \\
\hline
\end{tabular}

Fonte: O Autor.

A turbina apresentada na Fig. 2]é composta por três pás revestida com material plástico, onde para a fixação das mesmas, utilizam-se seis suportes: três inferiores e três superiores. Os mesmos se fixam em um tubo flange por meio de parafusos, que por sua vez está conectado ao rotor do gerador. Para a montagem no eixo central (1), são usados dois rolamentos capazes de receber cargas axiais e radiais, garantindo assim a rotação da turbina. Abaixo do tubo flange, está localizado o estator, com todas as bobinas dispostas axialmente. O estator é enroscado no eixo principal, podendo-se ajustar à distância entre os imãs e as bobinas de acordo com as alturas das mesmas.

Para a simulação utilizou-se apenas $1 / 3$ da geometria da turbina, devido a sua simetria axial. Com isso, houve uma redução no tempo de solução computacional 
sem alterar a precisão da análise. Além disso, alguns componentes que não eram alvo de investigação, como o eixo principal e rolamentos, foram supridos do modelo. As condições de operação dos rolamentos ainda são garantidas pela especificação do fabricante, onde o mesmo foi dimensionado para tal aplicação.

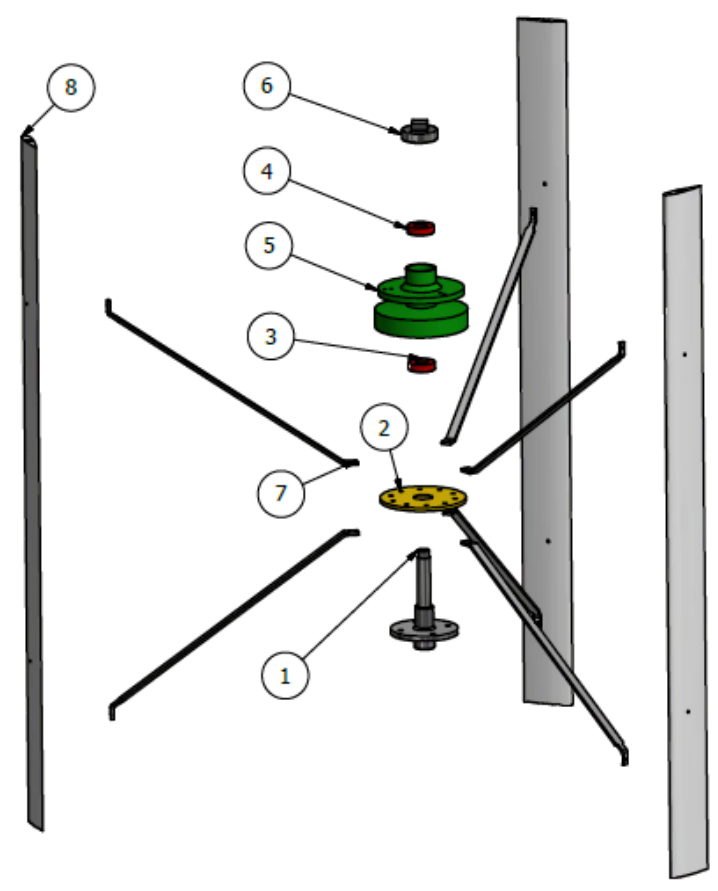

Figura 2: Principais componentes da Turbina eólica de eixo vertical Fonte: O Autor.

Após a finalização do modelo geométrico, o mesmo foi exportado para o Ansys (2012), software de simulação estrutural. Para tanto, e devido à uma malha computacional excessivamente numerosas em regiões na geometria com pequenos raios, foi necessária uma preparação adicional no software de CAD (INVENTOR 2016), onde foram retirados todos os pequenos raios na geometria. Para o material do gerador, optou-se pela escolha do nylon, que apresenta propriedades tal como densidade relativamente baixa (peso pequeno), boa resistência mecânica e considerável resistência à fadiga e à agentes químicos. Esse material foi utilizado em alguns componentes como o tubo flange e o estator. No eixo central, optou-se pelo aço 1045, onde o limite de resistência à ruptura é elevado, resultando em coeficientes de segurança estimados acima de valores exigidos em norma. O aço 1045 ainda tem vantagens como a fácil usinagem e o seu baixo custo (NORTON, 2013). $\mathrm{Na}$ simulação, foram empregadas condições de contato do tipo colado ("Bonded") entre os componentes. Esta condição de contato uni os corpos, de forma, que no decorrer da obtenção da solução no Ansys (2012), as fronteiras da geometria sejam respeitadas, evitando assim que as geometrias dos diversos componentes se interseccionem. Para a geração da malha, foi aplicado um elemento tetraédrico e um alto refino, a fim de garantir a continuidade na geometria, uma vez que os cálculos de tensão-deformação são adquiridos a partir dos deslocamentos dos elementos interconectados, denominados de "nós" (MARINHO, 2014), (LEITE; SHZU; MORAIS, 2012). A análise estática tem como objetivo principal quantificar a magnitude dos esforços internos e dos deslocamentos que se manifestam em qualquer sistema estrutural. O Ansys (2012) dispõe de uma gama de instrumentos para esse tipo de análise. No entanto, apenas alguns rucursos do software foram empregados. Conforme a Fig 3 , foram aplicadas a força gravitacional da terra, no valor de $9,81 \mathrm{~m} / \mathrm{s}^{2}$, a velocidade de rotação da turbina de 41,9 rad/s ou $400 \mathrm{rpm}$ e, ainda, superfícies fixas, limitando o deslocamento em todas as direções. Para simular apenas 1/3 da geometria, a condição de simetria axial foi aplicada nas fronteiras do tubo flange, limitando o deslocamento angular.

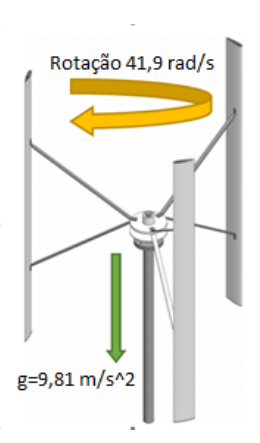

(a)

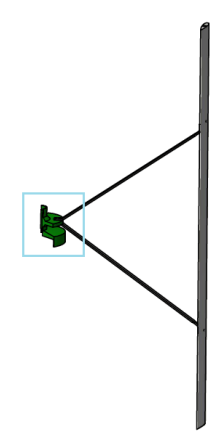

(b)

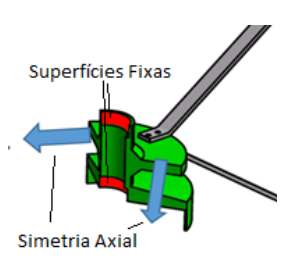

(c)

Figura 3: a) Vista Tridimensional da Turbina; b) Um terço da geometria a ser simulada; c) Detalhe da Imagem b Fonte: O Autor

Após algumas simulações de teste, foram propostas duas geometrias para os suportes das pás, ilustradas na Fig 4, onde o modelo A, Fig 4 a, mostra um suporte das pás com $5 \mathrm{~mm}$ de espessura e um raio de $7 \mathrm{~mm}$ para inclinação que faz contato com a pá. O modelo B, Fig $4 \mathrm{p}$, 
possue uma espessura de $6 \mathrm{~mm}$ e um raio de $37 \mathrm{~mm}$.

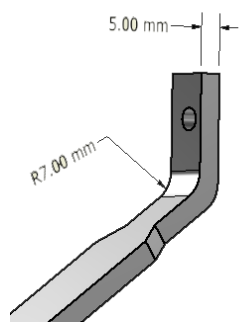

(a)

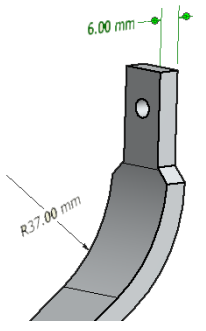

(b)
Figura 4: a) Modelo do suporte A; b) Modelo do suporte B Fonte: O Autor

A Fig 5 ilustra um dos resultados preliminares encontrados para o modelo B, onde é visualizado o campo de fator de segurança, ou seja, a relação entre a tensão encontrada e a tensão limite de ruptura do material.

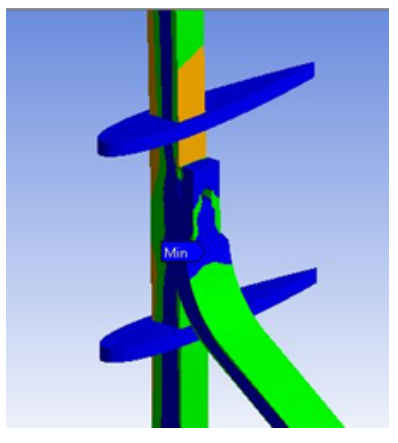

Figura 5: Ilustração do Fator de segurança mínimo no suporte B Fonte: O Autor

\section{RESULTADOS}

O dois modelos foram comparados em simulações envolvendo a fadiga do material, onde os mesmos foram submetidos à solicitações "cíclicas" de carregamento, conforme apresentado em Mott (2015). Os regimes de fadiga são definidos como: fadiga de baixo ciclo (FBC) e fadiga de alto ciclo (FAC), como apresentado em Norton (2013). Nesse sentido, a literatura apresenta uma divergência em relação ao número inicial de ciclos para a FAC, onde a partir do qual haja mais segurança em relação à falha por fadiga. Dowling (2012) sugere que a (FAC) tenha início em $10^{2}$ ciclos, já Yen e Fischer (1997) sugerem valores de $10^{3}$ até $10^{4}$ ciclos. O presente projeto propõe vida infinita à estrutura, logo, foi utilizado um número de ciclos de $10^{10}$. O software de simulação oferece uma gama de resultados, todavia, optou-se por obter apenas dados pertinentes a análise estrutural estática e por fadiga, afim de se obter uma análise das tensões atuantes na estrutura da turbina e uma estimativa de vida da mesma. As simulações realizadas com os dois modelos, obtiveram os resultados mostrados na Tab 2 .

Tabela 2: Resultados para os modelos A e B

\begin{tabular}{c|c|c}
\hline Modelos & A & B \\
\hline Deformação Máx. [mm] & 21,9 & 21,2 \\
\hline Tensão Máx. [MPa] & 202,6 & 179,7 \\
\hline Fator de Segurança Mín. & 1,38 & 1,56 \\
\hline
\end{tabular}
Fonte: O Autor.

Como observado na 2, o modelo B foi o que obteve melhores resultados, como deformação total e tensão equivalentes menores, e maior fator de segurança. Isto era esperado, visto que as regiões de maiores tensões estão localizadas próximas ao raio de inclinação do suporte. Existe uma alteração positiva do modelo A para o modelo B. O incremento de $1 \mathrm{~mm}$ na espessura, embora aumente a massa e o peso da estrutura, favorece a elevação da rigidez e do fator de segurança. Ambos os modelos foram comparados na viabilidade de construção, onde não tiveram diferenças significantes. Para evitar a concentração de tensão, é conveniente evitar grandes variações da seção transversal e utilizar um raio de transição grande Norton (2013). O modelo A apresentaria uma maior concentração de tensão, por isso, foi escolhido o modelo B para utilização do protótipo. Nas Figs. 6a e 6p é possível conferir que o ponto de menor fator de segurança, indicado pela seta vermelha, não se alterou entre os modelos, ou seja, continua sendo na dobra do suporte. Isto é, apenas com a alteração na geometria ganhou-se uma tensão admissível menor, gerando um fator de segurança maior no modelo B.

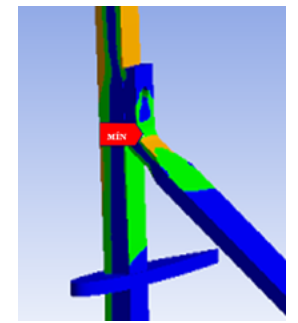

(a)

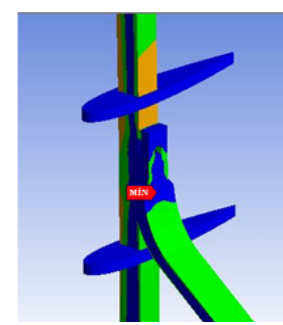

(b)
Figura 6: a) Ponto de menor fator de segurança do modelo A; (b) Ponto de menor fato de segurança do modelo B

Fonte: O Autor

Com os melhores resultados observados pelo modelo $\mathrm{B}$, o mesmo foi submetido à simulação cíclica de 
fadiga, onde como já mencionado, foi proposto vida infinita em alto ciclo. A resposta do teste foi positiva, já que se obteve estimativa mínima de $1,0381 \times 10^{10} \mathrm{ci}$ clos, como mostra a Fig. 7 A região da dobra do suporte continua recebendo a maior intensidade de tensão e sofrerá um maior efeito da fadiga. Ainda assim, é alcançada uma estimativa de vida infinita à estrutura.

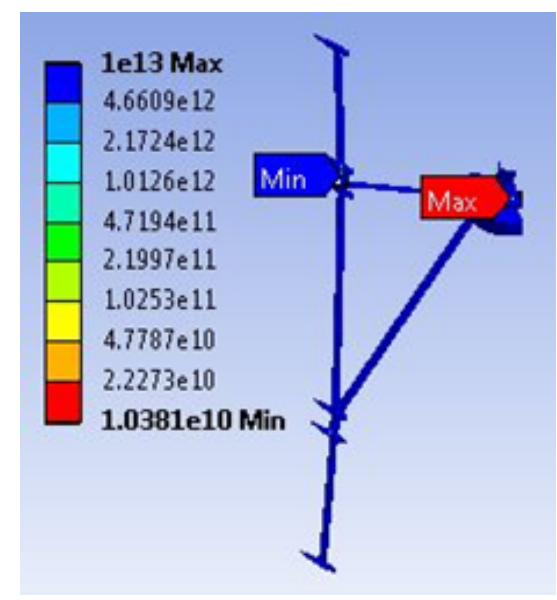

Figura 7: Resultados de simulação de fadiga para o Modelo B Fonte: O Autor

Nos modelos A e B buscou-se um fator de segurança maior que 1, dessa forma foi garantida as mínimas condições de segurança, mantendo a leveza da estrutura. Os resultados mostraram que com a mudança do raio na dobra do suporte, obteve-se uma deformação (máxima) menor no modelo B e ocorreu também um alívio na tensão máxima de 202,6 MPa para 179,7 MPa. Com isso, o fator de segurança aumentou de 1,38 no modelo A para 1,56 no modelo B, satisfazendo as condições mais satisfatórias de segurança. Com o modelo $\mathrm{B}$, também obtiveram-se resultados confiáveis no teste de fadiga, onde o número $1,0381 \times 10^{10}$ foi o mínimo de ciclos permitidos pela estrutura proposta. $\mathrm{O}$ método de elementos finitos utilizado, apresenta grau de confiabilidade satisfatório como mostrado em Awruch e Gomes (2014) e Awruch, Braun e Greco (2015). Assim como, a escolha do software ANSYS também foi correta visto que, possui um modelo de cálculo reconhecido e utilizado em Cesário et al. (2014), Toti, Netto Junior e Franco (2012) e Silva e Guidi (2012).

\section{CONCLUSÕES}

No presente trabalho foram analisados os esforços atuantes na estrutura da turbina eólica de eixo vertical, onde pode-se verificar regiões de máxima tensão e deformação. O modelo B por possuir raio de alivio maior, apresentou melhores resultados, como tensões mais baixas em relação ao modelo A e uma estimativa de vida infinita a estrutura. O projeto mostra-se viável, tendo em vista que o material utilizado não mostrou um custo elevado na construção de um protótipo para testes experimentais. Deste modo, trabalhos futuros de simulação com fluidos ou experimentais podem ser realizados no intuito de comparar e validar os resultados obtidos nesse trabalho.

\section{REFERÊNCIAS}

ANSYS. Product Launcher Release 12.0. 2012.

AWRUCH, A.; BRAUN, A.; GRECO, M. Engenharia do vento computacional e suas aplicações na engenharia civil. análise aerodinâmica e aeroelástica. Revista Internacional de Métodos Numéricos para Cálculo y Diseño en Ingeniería, Elsevier BV, v. 31, n. 1, p. 55-64, 12015.

AWRUCH, A.; GOMES, H. Análise da confiabilidade de estruturas de concreto armado com uma metodologia para inclusão de efeitos estocásticos de propriedades dos materiais. Revista Internacional de Métodos Numéricos para Cálculo y Diseño en Ingeniería, Elsevier BV, v. 30, n. 2, p. 85-96, 42014.

BRAGA, A. A.; PEREIRA DA SILVA, A.; CANZIAN, L. G.; KHALIL, R. V. Evolução da Tecnologia AALP. 2012. Disponível em: <https://evolucaoaalp. wordpress.com/category/evolucao-da-ciencia/>

CESÁRIO, A. A.; MATTOS, C.; LIMA, L. G. B. S.; TEIXEIRA, G. M.; DIESEL, F. N.; CASAS, W. FATIGUE ASSESSMENT OF a SEMI-TRAILER USING FE SPECTRAL ANALYSIS COMBINED WITH RANDOM FATIGUE CALCULATION. In: Proceedings of 10th World Congress on Computational Mechanics. : Editora Edgard Blucher, Ltda., 2014.

DOWLING, N. E. Mechanical Behavior of Materials. Englewood Cliffs, NJ: PRENTICE HALL, 2012. ISBN 0131395068. Disponível em: <http://www.ebook.de/de/product/16113867/norman e_dowling_mechanical_behavior_of_materials.html>

EPE. Balanço Energético Nacional. 2015. Disponível em: <https://ben.epe.gov.br/downloads/Relatorio Final_BEN_2015.pdf>. Acesso em: 1722017.

FAGAN, E.; FLANAGAN, M.; LEEN, S.; FLANAGAN, T.; DOYLE, A.; GOGGINS, J. Physical experimental static testing and structural design optimisation for a composite wind turbine blade. 
ANÁLISE ESTRUTURAL DE UMA TURBINA EÓLICA DE EIXO VERTICAL

Composite Structures, Elsevier BV, v. 164, p. 90-103, 32017 .

FARENZENA, A.; CARRAZEDO, R.; BAPTISTA

JUNIOR, I. P. Estudo de esforços atuantes na fundação de uma estrutura sob ação de ventos de alta intensidade. In: . Curitiba: , 2012. Disponível em: $<$ http://conferencias.utfpr.edu.br/ocs/index.php/sicite/ 2012/paper/viewFile/438/813>

HOWELL, R.; QIN, N.; EDWARDS, J.; DURRANI, $\mathrm{N}$. Wind tunnel and numerical study of a small vertical axis wind turbine. Renewable Energy, Elsevier BV, v. 35, n. 2, p. 412-422, 22010.

INVENTOR. Product Launcher Release 2016. 2016.

KENNEDY, S. Wind power planning: assessing long-term costs and benefits. Energy Policy, Elsevier BV, v. 33, n. 13, p. 1661-1675, 92005.

LEANDRO FILHO, F; SILVA JUNIOR, F. I.; DUARTE JUNIOR, O. Avaliação de turbinas eólicas utilizando simulaçẽs estocásticas monte carlo. In: . São Luiz: , 2012. Disponível em: <http://www.abcm.org. br/anais/conem/2012/PDF/CONEM2012-0367.PDF>

LEITE, F. A.; SHZU, M. A. M.; MORAIS, M. V. G. de. Análise estrutural de uma válvula de escape de um motor à combustão interna através do método dos elementos finitos(mef). In: . São Luiz: , 2012. Disponível em: <http://www.abcm.org.br/anais/ conem/2012/PDF/CONEM2012-1733.PDF>

LI, Q.; MAEDA, T.; KAMADA, Y.; MURATA, J.; YAMAMOTO, M.; OGASAWARA, T.; SHIMIZU, K.; KOGAKI, T. Study on power performance for straight-bladed vertical axis wind turbine by field and wind tunnel test. Renewable Energy, Elsevier BV, v. 90, p. 291-300, 52016.

MARINHO, I. J. P. PROJETO ÓTIMO DE ESTRUTURAS METÁLICAS DE ARQUIBANCADAS REUTILIZÁVEIS VIA ANSYS. Tese (Doutorado), Rio de Janeiro, 2014.

MARTINS, F.; GUARNIERI, R.; PEREIRA, E. O aproveitamento da energia eólica. Revista Brasileira de Ensino de Física, FapUNIFESP (SciELO), v. 30, n. 1, p. 13041-13043, 2008.

MOTT, R. L. Elementos de Máquina em Projetos Mecânicos. Pearson, 2015. ISBN 9788543005904. Disponível em: <https://www.amazon.com/ Elementos-Maquina-em-Projetos-Mecanicos/ dp/8543005906\%3FSubscriptionId\%

3D0JYN1NVW651KCA56C102\%26tag\%
3Dtechkie-20\%26linkCode\%3Dxm2\%26camp\%
3D2025\%26creative\%3D165953\%26creativeASIN\%
3D8543005906>

NORTON, R. L. Projeto de Máquinas: Uma Abordagem Integrada (Portuguese Edition). Bookman, 2013. ISBN 9788582600221. Disponível em: <https://www.amazon.com/Projeto-M\%C3\% A1quinas-Abordagem-Integrada-Portuguese-ebook/ dp/B016LFWKIM\%3FSubscriptionId\% 3D0JYN1NVW651KCA56C102\%26tag\% 3Dtechkie-20\%26linkCode\%3Dxm2\%26camp\% 3D2025\%26creative\%3D165953\%26creativeASIN\% 3DB016LFWKIM>.

SILVA, F. de A.; GUIDI, E. S. Análise da concentração de tensões em parafusos de fixaçãção através de um procedimento numérico-experimental. In: . São Luiz: , 2012. Disponível em: <http://www.abcm.org.br/anais/ conem/2012/PDF/CONEM2012-1463.PDF>

TOTI, F. de A.; NETTO JUNIOR, A. V.; FRANCO, S. M. Análise da concentração de tensão na alteração de seção de eixo escalonado utilizando sistema cae. In: . 2012. Disponível em: <http://www.abcm.org.br/ anais/conem/2012/PDF/CONEM2012-0455.PDF>

YEN, B.; FISCHER, J. Structural details, and discontinuities in steel safety and reliability of metal structures. ASCE, 1997. 